\title{
Condición física y perfil antropométrico de atletas de artes marciales mixtas
}

Bruno Ferreira Marinho일 Fabrício Boscolo Del Vecchio ${ }^{1,2}$, Emerson Franchini ${ }^{1}$

${ }^{1}$ Martial Arts and Combat Sports Research Group, School of Physical Education and Sport, Universidade de São Paulo (Brasil). ${ }^{2}$ Universidade Federal de Pelotas (Brasil)

Recibido 02/08/2011 - A ceptado 07/09/2011

\section{Resumen}

Objetivo: el propósito de este estudio es identificar la condición física y el perfil antropométrico de atletas de artes marciales mixtas (MMA) y las correlaciones entre estas variables. Sujetos y método: Participaron en el estudio trece atletas varones de M MA (30 \pm 4 años). Se tomaron medidas antropométricas y realizaron los siguientes test: flexitest adaptado, abdominales, flexiones de brazos, detente horizontal, flexión de brazos en suspensión en barra, y una repetición máxima (1RM) de press de banca y sentadilla. Resultados: los principales resultados son los siguientes: masa corporal (kg): $82.1 \pm 10.9$; grasa corporal (\%): $11.87 \pm 5.11$; flexibilidad (puntuación): $18.38 \pm 4.07$; abdominales (repeticiones): $43 \pm 11$; flexiones de brazos (repeticiones): $41 \pm 9$; detente horizontal (m): $2.19 \pm 0.25$; flexión de brazos en suspensión en barra (s): $34 \pm 11$, 1RM press de banca $(\mathrm{kg})$ : $76 \pm 23$; 1 RM sentadilla $(\mathrm{kg}): 73 \pm 15$. Además, observamos correlaciones significativas entre variables antropométricas y condición física: grasa corporal y detente horizontal $(R=-0.75)$; grasa corporal y flexión de brazos en suspensión en barra $(R=$ $-0.67)$; altura y $1 R M$ sentadilla $(R=0.67)$; circunferencia de brazo y $1 R M$ press de banca $(R=0.77)$. Conclusión: Los atletas de MMA participantes en esta investigación muestran un pobre rendimiento muscular. La grasa corporal se correlacionó negativamente con el rendimiento en potencia y con una fuerte resistencia, mientras que la circunferencia del brazo se relaciona positivamente con la fuerza máxima de la parte superior del cuerpo.

Palabras clave: artes marciales mixtas, deportes de combate, valoración física.

Physical fitness and anthropometric profile of mixed martial arts athletes

Abstract: Objective: The aim of this study was to identify the physical fitness and anthropometric profile of mixed martial arts (MMA) athletes and the correlations between these variables. Subjects and methods: Thirteen male MMA athletes (30 \pm 4 years-old) participated in this study. They were submitted to anthropometric measurements and the following tests: adapted flexitest, sit-ups, push-ups, long jump, flexed arm hang, 1RM bench press and squat. Results: Main results are as follows: body mass (kg): $82.1 \pm 10.9$; body fat (\%): $11.87 \pm 5.11$; flexibility (score): $18.38 \pm 4.07$; sit-ups (rep): $43 \pm 11$; push- 
ups (rep): $41 \pm 9$; long jump (m): $2.19 \pm 0.25$; flexed arm hang (s): $34 \pm 11$, 1RM benchpress $(\mathrm{kg}): 76 \pm 23$; $1 \mathrm{RM}$ squat $(\mathrm{kg}): 73 \pm 15$. Furthermore we observed results showed significant correlations between anthropometric variables and physical fitness: body fat and long jump ( $R=-0.75)$; body fat and flexed arm hang $(R=-0.67)$; height and squat $\operatorname{IRM}(R=0.67)$; arm circumference and bench press $1 R M(R=0.77)$. Conclusion: MMA athletes involved in this investigation have showed poor neuromuscular performance. Body fat was negatively correlated with both power and strength endurance performance, while arm circumference was positively related to upper body maximum strength.

Key words: mixed martial arts, combat sports, physical assessment.

\section{Perfil antropométrico e motor de atletas de mixed martial arts}

Resumo: Objetivo: 0 objetivo desse estudo foi determinar o perfil antropométrico e motor de atletas de mixed martial arts (MMA) e a correlação entre essas variáveis. Sujeitos e métodos: Treze atletas de MMA ( $30 \pm 4$ anos de idade) do sexo masculino participaram desse estudo, e foram submetidos a medidas antropométricas e aos testes: flexiteste adaptado, abdominais, flexões de braço, suspensão na barra, salto horizontal e 1RM no supino reto e agachamento. Resultados: Os principais resultados foram: massa corporal (kg): $82.1 \pm 10.9$; \%G: $11.87 \pm 5.11$; flexibilidade (pontos): $18.38 \pm 4.07$; abdominais (rep): $43 \pm 11$; flexões de braço (rep): $41 \pm 9$; suspensão na barra (s): $34 \pm 11$; salto horizontal (m): $2.19 \pm 0.25 ; 1 R M$ no supino reto $(\mathrm{kg}): 76 \pm 23 ; 1 R M$ no agachamento $(\mathrm{kg}): 73$ \pm 15 . Além disso, os resultados apresentaram correlações significantes entre variáveis antropométricas e aptidão física: \%G e salto horizontal ( $r=-0.75) ; \% G$ e suspensão na barra ( $r=-0.67)$; estatura e 1RM no agachamento ( $r=0.67)$; circunferência do braço e 1RM no supino ( $r=0.77$ ). Conclusão: $0 s$ atletas de M M A envolvidos no presente estudo apresentaram baixo rendimento neuromuscular. A gordura corporal apresentou correlação negativa com o desempenho de potência e resistência muscular, enquanto a circunferência do braço esta relacionada positivamente com a força máxima de membros superiores.

Palavras-chave: artes marciais mistas, esportes de combate, avaliação física. 


\section{Condición física y perfil antropométrico de atletas de artes marciales mixtas}

Bruno Ferreira Marinho', Fabrício Boscolo Del Vecchio $0^{1,2}$, Emerson Franchini ${ }^{1}$

${ }^{1}$ Martial Arts and Combat Sports Research Group, School of Physical Education and Sport, Universidade de São Paulo (Brasil). ${ }^{2}$ Universidade Federal de Pelotas (Brasil)

\section{Introducción}

Las artes marciales mixtas (mixed martial arts - MMA) es un deporte que integra acciones motrices de diferentes deportes de combate tales como boxeo, kick-boxing, muay thai, jiu-jitsu, judo y lucha, dando como resultado un amplio espectro de técnicas (Del Vecchio, et al., 2011). Además, los atletas que tienen un mayor éxito en esta disciplina combinan habilidades de nivel de elite con extraordinarios niveles de fuerza y acondicionamiento (Amtmann, 2004).

La literatura en deportes de combate muestra diversas investigaciones sobre las características físicas y fisiológicas de judo (Franchini, et al., 2011; Franchini, et al., 2007), lucha (Horswill, et al., 1992; Mirzaei, et al., 2009; Yoon, 2002), jiu-jitsu brasileño (Andreato, et al., 2011), kárate (Doria, et al., 2009; Probst, et al., 2007), taekwondo (Heller, et al., 1998; Toskovic, et al., 2004), kung-fu (Artioli, et al., 2009), kick-boxing y muay-thai (Silva, et al., 2011). Aunque existen algunos estudios relativos a las respuestas fisiológicas al entrenamiento y combate de MMA (Amtmann, et al., 2008) y a la estructura temporal de los combates de MMA (Del Vecchio, et al., 2011), únicamente hemos encontrado dos estudios relativos a la condición física de estos atletas (Schick, et al., 2010; Braswell, et al., 2010).

Estudios previos que investigaron la condición física de atletas de otros deportes tales como el judo (Franchini, et al., 2011), lucha (M irzaei, et al., 2009; Yoon, 2002), kung-fu (Artioli, et al., 2009), kárate (Roschel, et al., 2009) y jiujitsu (Andreato, et al., 2011), mostraron grandes niveles de fuerza isométrica y máxima, flexibilidad, potencia muscular y resistencia muscular. Estas variables son específicas de acuerdo a la modalidad. En deportes tipo grappling (sujección) se necesitan altos niveles de resistencia muscular, fuerza isométrica y flexibilidad para inmovilizar o lograr la sumisión del oponente. En deportes de golpeo la fuerza máxima y la potencia muscular son fundamentales para puntuar o poner fuera de combate al oponente mediante puñetazos y patadas. 
Como se ha señalado, se asume que las MMA implican diferentes tipos de acciones motrices (Del Vecchio, et al., 2011), y por tanto sería interesante conocer la condición física de los luchadores de MMA, y así poder compararla con luchadores de otros deportes de combate. Además, la determinación del perfil antropométrico y motor de estos atletas es importante para definir qué aspectos de la condición física son relevantes para el deporte, y que esta información pueda ser utilizada para monitorizar y planificar mejor el entrenamiento de la condición física, y consecuentemente que estos atletas tengan mayores posibilidades de lograr el éxito competitivo (A mtmann, 2010; Aruga, et al., 2003; Franchini, et al., 2011). Por tanto, este estudio tuvo por objetivo identificar la condición física y el perfil antropométrico de atletas de MMA y las correlaciones entre estas variables.

\section{Material y método}

- Muestra

Participaron en el estudio 13 atletas varones practicantes de MMA. Estos atletas entrenaban MMA regularmente durante cinco días por semana y tenían cuatro años de experiencia en el entrenamiento del deporte, en competiciones de nivel nacional. Previamente a su participación, los participantes fueron informados sobre la naturaleza y riesgos que implicaba el experimento, así como sobre los procedimientos para los test. Todos ellos firmaron una declaración por escrito de consentimiento informado. Todos los atletas respondieron negativamente a todos los ítems del cuestionario PAR-Q ${ }^{1}$ (Shephard, 1988).

- Rutina de test

Los test fueron realizados en dos días consecutivos, con una separación de 24 horas. Durante el primer día se tomaron las medidas antropométricas para estimar la composición corporal, y se aplicaron los test de flexibilidad y fuerza resistencia. Posteriormente, se midieron la fuerza máxima y la potencia muscular. Los test para verificar la condición física fueron aplicados en el siguiente orden: flexitest adaptado, abdominales, flexiones de brazos, flexión de brazos en suspensión en barra, press de banca y sentadilla 1RM, y detente horizontal.

- Medidas antropométricas

Todos los procedimientos se realizaron de acuerdo con las recomendaciones de la International Society for Advancement in Kinanthropometry (ISAK). Se tomaron medidas de la masa corporal $(\mathrm{kg})$ y altura $(\mathrm{cm})$ de los atletas mediante una báscula con estadiómetro W elmy (precisión de $0.5 \mathrm{~cm}$ y $100 \mathrm{~g}$ respectivamente). Como resultado de estas medidas se obtuvo el índice de masa corporal (IMC, en $\mathrm{kg} / \mathrm{m}^{2}$ ). Para estimar la composición corporal, se midieron los pliegues cutáneos de abdomen, tríceps y subescapular. Cada uno de estos elementos fue medido tres veces -se tomó como referente el valor medio- en el hemicuerpo derecho, en

1 El Physical activity readiness questionnaire [Cuestionario sobre el estado de preparación para la actividad física] (PAR-Q) es un cuestionario que trata de determinar si la práctica de ejercicio físico es segura o puede presentar algunos riesgos para el individuo, a través de diversas preguntas sobre su historial de salud. 
rotación, utilizando un calibre CESCORF ${ }^{\circledR}$ (Brazil). La grasa corporal se estimó mediante la ecuación propuesta por Lohman (1992), que es la designada por la National Collegiate Athletic Association (NCAA) (Clark, et al., 2002).

- Flexibilidad

La flexibilidad fue medida mediante el flexitest adaptado (Araújo, 2004). Consideramos ocho movimientos, desde el hemicuerpo derecho, cadera, tronco y hombro.

- Resistencia muscular

Se utilizó un test de flexiones para evaluar la fuerza resistencia de la parte superior del cuerpo y un test de abdominales en un minuto para evaluar la fuerza resistencia de los abdominales. Para ambos test se siguieron las indicaciones propuestas por Pollock, et al. (1990).

- Potencia muscular

Para evaluar la potencia de la parte inferior del cuerpo se utilizó el test de detente horizontal. Cada atleta realizó tres intentos, registrándose la distancia que el atleta lograba desde la primera marca hasta el calcáneo (Johnson, et al., 1979). Para el análisis se consideró el mejor de estos tres saltos.

- Fuerza máxima

Se evaluó una repetición máxima (1RM) de press de banca y sentadilla. Todos los atletas estaban familiarizados con ambos ejercicios. Cada atleta realizó entre 3 y 5 intentos, con intervalos de 3 a 5 minutos entre ellos, siguiendo las recomendaciones realizadas por Brown y W eir (Brown, et al., 2001).

- Fuerza isométrica

La fuerza resistencia de la parte superior del cuerpo fue evaluada utilizando el test de flexión de brazos en suspensión en barra, en la que cada atleta permaneció suspendido con la barbilla sobre la barra tanto como le fue posible (Johnson $\&$ Nelson, 1979). Se registró el tiempo en segundos.

- Análisis estadístico

Los resultados se presentan como media \pm desviación estándar y valores mínimos y máximos. Se utilizó el coeficiente de correlación de Spearman para calcular las correlaciones entre variables, puesto que los datos no presentaban una distribución normal. El análisis estadístico se realizó mediante el software estadístico Sigma-Plot - 11.0 estableciendo el nivel de significancia en todos los casos en $p<0.05$.

\section{Resultados}

Las características antropométricas detalladas de los atletas de MMA se presentan en la Tabla 1, mientras que los resultados de los test neuromusculares aplicados se muestran en la Tabla 2. 
Tabla 1. Características antropométricas de los atletas de artes marciales mixtas $(n=13)$.

\begin{tabular}{lccc}
\hline Variable & Media \pm DS & Mínimo & Máximo \\
\hline Edad (años) & $30.0 \pm 4.4$ & 24 & 37 \\
Masa corporal $(\mathrm{kg})$ & $82.1 \pm 10.9$ & 63.2 & 98.5 \\
Altura $(\mathrm{cm})$ & $176.0 \pm 0.05$ & 168 & 187 \\
IMC $\left(\mathrm{kg} / \mathrm{m}^{2}\right)$ & $26.41 \pm 3.49$ & 20.4 & 30.7 \\
Grasa corporal $(\%)$ & $11.87 \pm 5.11$ & 4.9 & 22.1 \\
Circunferencia de brazo (cm) & $33.46 \pm 3.0$ & 29 & 38 \\
$\quad$ & & \\
Gríceps & & & 28.6 \\
Subescapular de pliegues $(\mathrm{mm})$ & $8.93 \pm 6.74$ & 3.5 & 20.2 \\
Abdominal & $13.95 \pm 4.85$ & 6.6 & 30.2 \\
\hline
\end{tabular}

IMC: índice de masa corporal; DS: desviación estándar.

Tabla 2. Condición física de los atletas de artes marciales mixtas $(n=13)$.

\begin{tabular}{lccc}
\hline Variable & Media \pm DS & Mínimo & Máximo \\
\hline Flexitest (puntuación) & $18.38 \pm 4.07$ & 14 & 26 \\
Abdominales (rep.) & $43 \pm 11$ & 24 & 65 \\
Flexiones (rep.) & $41 \pm 9$ & 25 & 55 \\
Detente horizontal $(\mathrm{m})$ & $2.19 \pm 0.25$ & 1.94 & 2.93 \\
Press de banca 1RM $(\mathrm{kg})$ & $76 \pm 23$ & 40 & 110 \\
Press de banca 1RM (kg/kg de masa corporal) & $0.93 \pm 2.07$ & 0.63 & 1.12 \\
Sentadilla 1RM (kg) & $73 \pm 15$ & 60 & 110 \\
Sentadilla 1RM (kg/kg de masa corporal) & $0.89 \pm 1.38$ & 0.95 & 1.12 \\
Flexión de brazos en suspensión en barra $(\mathrm{s})$ & $34 \pm 11$ & 16 & 46 \\
\hline
\end{tabular}

1RM: 1 repetición máxima; DS: desviación estándar.

En relación a las correlaciones, la grasa corporal se relacionó negativamente con la potencia muscular de la parte inferior del cuerpo ( $r=-0.75 ; p<0.05)$ y con la fuerza resistencia $(r=-0.67 ; p<0.05)$, mientras que la altura se relacionó positivamente con la fuerza máxima de la parte inferior del cuerpo $(r=0.67$; $p<$ 0.05) y la circunferencia de brazo se relacionó positivamente con la fuerza máxima de la parte superior del cuerpo $(r=0.77 ; p<0.05)$.

\section{Discusión}

Este estudio, que tuvo el propósito de evaluar la antropometría y condición física de atletas de MMA, observó que su IMC (26.41 $\left.\pm 3.49 \mathrm{~kg} / \mathrm{m}^{2}\right)$ se sitúa en el sobrepeso, de acuerdo a la World Health Organization [Organización Mundial de la Salud] (2010). Aunque el IMC no es un buen indicador de la composición corporal para los atletas, puesto que poseen una elevada cantidad de masa magra, los valores son más altos que aquellos que se encuentran en judo (Rouveix, et al., 2007) y kárate (Sbriccoli, et al., 2010). 
El porcentaje de grasa corporal ha mostrado ser un aspecto relevante en los deportes de combate, especialmente porque los atletas de estas modalidades son clasificados de acuerdo a su peso corporal. Una menor grasa corporal es una de las características de composición corporal que diferencia a los atletas de élite de aquellos que no lo son (Giampietro, et al., 2003; Schick, et al., 2010). Los datos obtenidos en este estudio son similares a los de luchadores de MMA (Schick, et al., 2010) y de otros deportes de combate de control, como el judo (Franchini, et al., 2011; Franchini, et al., 2007), lucha (Mirzaei, et al., 2009; Schmidt, et al., 2005) y jiu-jitsu brasileño (Sterkowicz-Przybycie, 2010). Cuando se comparan con deportes de combate de golpeo, como el kárate (Milanez, et al., 2010) y kung-fu (Artioli, et al., 2009), los valores obtenidos en este estudio fueron mayores. En deportes de combate de control, como la lucha, los atletas tratan de maximizar la cantidad de tejido magro, minimizar la cantidad de grasa corporal, y minimizar el peso corporal total (Yoon, 2002). Así, muchos atletas de deportes de combate reducen su grasa corporal para competir en categorías de peso inferior, con el objeto de maximizar su fuerza relativa (Franchini, et al., 2011).

Estos atletas presentan un alto nivel de condición física, especialmente flexibilidad y fuerza resistencia. No obstante, aunque los atletas en este estudio han presentado un alto nivel de flexibilidad, no tenemos un referente de comparación. Sin embargo, comparando luchadores con mayor y menor éxito, Yoon (2002) señaló que la flexibilidad podría ser una variable discriminante. Para luchadores universitarios, las medidas del test sit-and-reach eran mayores en el grupo con éxito que en el grupo de luchadores de éxito moderado y menor (Yoon, 2002). La literatura refiere mayores niveles de flexibilidad en judo (Little, 1991; Thomas, et al., 1989) y jiu-jitsu brasileño (Andreato, et al., 2011). En jiu-jitsu brasileño, e.g., la articulación de la cadera tiene una gran demanda durante el combate. Por tanto, menores niveles de flexibilidad en esta articulación puede disminuir el rendimiento en jiu-jitsu (Andreato, et al., 2011).

El perfil de condición física de luchadores de MMA (Schick, et al., 2010) mostró similares características al compararse al de judokas y luchadores de élite. Aunque un estudio previo no observó diferencias entre la condición física de luchadores de MMA y practicantes de kárate (Braswell, et al., 2010). En relación al judo (Sertic, et al., 2006), por ejemplo, los atletas acumularon $58 \pm 6$ repeticiones en el test de abdominales, y $53 \pm 7$ los atletas de taekwondo (Toskovic, et al., 2002). En la prueba de flexiones, los atletas de judo alcanzaron las 72 repeticiones en un minuto (Taylor, et al., 1981). En deportes de combate, la mayoría de estudios sobre fuerza resistencia han evaluado esta capacidad utilizando los test de abdominales y de flexión de brazos (Andreato, et al., 2011; Franchini, et al., 2011; Mirzaei, et al., 2009).

El test de una repetición máxima (1RM) es utilizado habitualmente por los entrenadores para medir la carga máxima (Braith, et al., 1993). En el test de press de banca $1 R M$, el rendimiento fue similar al de los atletas de kárate brasileños, con $76.3 \pm 16.8 \mathrm{~kg}$ (Roschel, et al., 2009), pero inferior al de los atletas de judo (Fagerlund, et al., 1991; Franchini, et al., 2007; Thomas, et al., 1989), y al de atletas japoneses de kárate altamente entrenados, con $87 \pm 12.5 \mathrm{Kg}$ (Imamura, et al., 1998). En el test de sentadilla, los atletas de kárate (Roschel, et al., 2009) y 
el equipo brasileño de judo (Franchini, et al., 2007) presentaron valores más altos (128.6 $\pm 20.4 \mathrm{~kg}$ y $104 \pm 27 \mathrm{~kg}$, respectivamente). Valores de referencia derivados de la evaluación de más de 160 judokas de alto nivel competitivo (Aruga, et al., 2003) clasificarían a estos atletas como débiles en press de banca y sentadilla. Para ser considerados como aceptables, los resultados deberían estar alrededor de 102.5 a $117.5 \mathrm{~kg}$ y 127.5 a $145 \mathrm{~kg}$, respectivamente (Aruga, et al., 2003). En valores relativos ( $\mathrm{kg} / \mathrm{kg}$ de masa corporal), estos resultados fueron más bajos que los valores encontrados en judo (Franchini, et al., 2007), MMA (Schick, et al., 2010) y lucha (Mirzaei, et al., 2009). En relación al detente horizontal, los atletas participantes en esta investigación obtuvieron valores inferiores a los de atletas de judo, que obtuvieron los resultados de $2.37 \pm 19.8 \mathrm{~m}$ (Bratic, et al., 2008).

La fuerza isométrica es un componente relevante de la condición física de los deportes de combate. En lucha, trabajos previos han demostrado que la fuerza isométrica llega a comprometerse tanto en un combate como a lo largo de un torneo (Kraemer, et al., 2001; Kraemer, et al., 2004), indicando que esta variable está altamente solicitada durante el combate. Aunque los atletas en este estudio han mostrado un alto nivel de fuerza isométrica, medida a través del test de flexión de brazos en suspensión en barra, no se han encontrado valores de referencia.

Aunque los datos sobre correlaciones han de tomarse con precaución, los resultados muestran la importancia de un bajo porcentaje de grasa corporal, puesto que se correlaciona negativamente con la potencia de las extremidades inferiores y con la fuerza resistencia isométrica de la parte superior del cuerpo. Los estudios que verifican la condición física en atletas universitarios de judo de alto nivel (Franchini, et al., 2005) y en atletas del equipo brasileño de kárate (Roschel, et al., 2009), observaron una influencia negativa de la grasa corporal en el rendimiento motor.

La altura se correlacionó positivamente con la sentadilla 1RM, indicando que los atletas más altos tienen una mayor habilidad para producir fuerza en las piernas. La circunferencia de brazo mostró una fuerte correlación con la fuerza de la parte superior del cuerpo, señalando la necesidad de incrementar la masa muscular en este segmento corporal para así incrementar los niveles de fuerza de la parte superior del cuerpo. Con todo, han de realizarse más estudios para investigar la contribución de la condición física al rendimiento en el combate de MMA.

\section{Conclusión}

Los atletas de MMA que tomaron parte en esta investigación presentaron un pobre perfil neuromuscular, aunque el porcentaje de grasa corporal era similar al que presentan atletas de otros deportes de combate. En este grupo, las diferentes manifestaciones de fuerza se correlacionaron con medidas antropométricas, especialmente porcentaje de grasa corporal, altura y circunferencia de brazo. 


\section{REFEREN CIAS}

Amtmann, J. A. (2004). Self-reported training methods of mixed martial artists at a regional reality fighting event. The Journal of Strength and Conditioning Research, 18(1), 194-196.

Amtmann, J. A., Amtmann, K. A., \& Spath, W. K. (2008). Lactate and rate of perceived exertion responses of athletes training for and competing in a mixed martial arts event. Journal of Strength and Conditioning Research, 22(2), 645-647.

Amtmann, J. (2010). High intensity training and the Mixed Martial Artist. New York: N ova Science.

Andreato, L. V., Moraes, S. M. F., Gomes, T. L. M., Esteves, J. V. C., Andreato, T. V., \& Franchini, E. (2011). Estimated aerobic power, muscular strength and flexibility in elite Brazilian Jiu-jitsu athletes. Science and Sports, (en prensa).

Araújo, C. G. S. (2004). Flexitest: an innovative flexibility assessment method. United States: Human Kinetics.

Artioli, G. G., Gualano, B., Franchini, E., Batista, R. N., Polacow, V. O., \& Lancha, A. H., Jr. (2009). Physiological, performance, and nutritional profile of the Brazilian Olympic W ushu (kung-fu) team. The Journal of Strength and Conditioning Research, 23(1), 20-25.

Aruga, S., Onda, T., Aso, K., Yamashita, Y., Nakanishi, H., Shirase, $H_{\text {., }}$ et al. (2003). Measurement of barbell lifting capacity and making strength standards in judo players. Tokai Journal of Sports Medical Science, 15, 7-17.

Braith, R. W., Graves, J. E., Leggett, S. H., \& Pollock, M. L. (1993). Effect of training on the relationship between maximal and submaximal strength. Medicine and Science in Sports and Exercise, 25(1), 132-138.

Braswell, M., Szymanski, D., Szymanski, J., Dixon, E., Gilliam, S., W ood, R., et al. (2010). Physiological Differences In Mixed Martial Artist And Traditional Martial Artists: A Pilot Study. The Journal of Strength and Conditioning Research, 24, 1.

Bratic, M., Radovanovic, D., \& N urkic, M. (2008). The effects of preparation period training program on muscular strenght of first-class judo athletes. A cta Medica Medianae, 46(4), 22-26.

Brown, L., \& Weir, J. (2001). ASEP procedures recommendation I: accurate assessment of muscular strength and power. Journal of Exercise Physiology, 4(3), 1-21.

Clark, R. R., Oppliger, R. A., \& Sullivan, J. C. (2002). Cross-validation of the NCAA method to predict body fat for minimum weight in collegiate wrestlers. Clinical Jounal of Sport M edicine, 12(5), 285-290.

Del Vecchio F. B., Hirata S, \& Franchini E. (2011). A review of time-motion analysis and combat development in mixed martial arts matches at regional level tournaments. Perceptual and Motor Skills, 112(2), 639-648. 
Doria, C., Veicsteinas, A., Limonta, E., Maggioni, M. A., Aschieri, P., Eusebi, F., et al. (2009). Energetics of karate (kata and kumite techniques) in top-level athletes. European Journal of Applied Physiology, 107(5), 603-610.

Fagerlund, R., \& Hakkinen, H. (1991). Strength profile of finnish judoists-measurement and evaluation. Biology of Sport, 8(03), 143-149.

Franchini, E., Del Vecchio, F. B., Matsushigue, K. A., \& Artioli, G. G. (2011). Physiological profiles of elite judo athletes. Sports M edicine, 41(2), 147-166.

Franchini, E., Nunes, A. V., Moraes, J. M., \& Del Vecchio, F. B. (2007). Physical fitness and anthropometrical profile of the Brazilian male judo team. Journal of Physiological Anthropology, 26(2), 59-67.

Franchini, E., Takito, M. Y., \& Bertuzzi, R. (2005). Morphological, physiological and technical variables in high-level college judoists. Archives of Budo, $1,1-7$.

Giampietro, M., Pujia, A., \& Bertini, I. (2003). Anthropometric features and body composition of young athletes practicing karate at a high and medium competitive level. Acta Diabetologica, 40(1), 145-148.

Heller, J., Peric, T., Dlouha, R., Kohlikova, E., Melichna, J., \& N ovakova, H. (1998). Physiological profiles of male and female taekwon-do (ITF) black belts. Jounal of Sports Sciences, 16(3), 243-249.

Horswill, C. A., Miller, J. E., Scott, J. R., Smith, C. M., W elk, G., \& Van Handel, P. (1992). Anaerobic and aerobic power in arms and legs of elite senior wrestlers. International Journal of Sports Medicine, 13(8), 558-561.

Imamura, H., Yoshimura, Y., Uchida, K., N ishimura, S., \& Nakazawa, A. (1998). Maximal oxygen uptake, body composition and strength of highly competitive and novice karate practitioners. Applied Human Science, 17(5), 215-218.

Johnson, B. L., \& Nelson, J. K. (1979). Practical M easurements for Evaluation in Physical Education. Minnesota: Burgess.

Kraemer, W. J., Fry, A. C., Rubin, M. R., Triplett-McBride, T., Gordon, S. E., Koziris, L. P., Lynch, J. M., Volek, J. S., Meuffels, D. E., Newton, R. U., $\&$ Fleck, S. J. (2001). Physiological and performance responses to tournament wrestling. Medicine and Science in Sports and Exercise, 33(8), 1367-1378.

Kraemer, W. J., Vescovi, J. D., \& Dixon, P. (2004). The physiological basis of wrestling: Implications for conditioning programs. Strength and Conditioning Journal, 26(2), 10-15.

Krstulovic, S., Zuvela, F., \& Katic, R. (2006). Biomotor systems in elite junior judoists. Collegium Antropologicum, 30(4), 845-851.

Little, N. G. (1991). Physical performance attributes of junior and senior women, juvenile, junior, and senior men judokas. Journal of Sports Medicine and Physical Fitness, 31(4), 510-520.

Lohman, T. G. (1992). Advances in body composition assessment. Canada: Human Kinetics 
Milanez, V., Lima, M.S, Gobatto, C., Perandini, L., Nakamura, F., \& Ribeiro, L. (2010). Correlates of session-rate of perceived exertion (RPE) in a karate training session. Science and Sports, 26(1), 38-43.

Mirzaei, B., Curby, D. G., Rahmani-N ia, F., \& Moghadasi, M. (2009). Physiological profile of elite Iranian junior freestyle wrestlers. Journal of Strength and Conditioning Research, 23(8), 2339-2344.

Pollock, M. L., W ilmore, J. H., \& Fox, S. M. (1990). Exercise in health and disease: evaluation and prescription for prevention and rehabilitation ( $2 \mathrm{a}$ ed.). Philadelphia: Saunders

Probst, M. M., Fletcher, R., \& Seelig, D. S. (2007). A comparison of lower-body flexibility, strength, and knee stability between karate athletes and active controls. Journal of Strength and Conditioning Research, 21(2), 451-455.

Roschel, H., Batista, M., Monteiro, R., Bertuzzi, R., Barroso, R., Loturco, I., Ugrinowitsch, C., Tricoli, V., \& Franchini, E. (2009). Association between neuromuscular tests and kumite performance on the Brazilian Karate $N$ ational Team. Journal of Sports Science Medicine, 8(3), 20-24.

Rouveix, M., Bouget, M., Pannafieux, C., Champely, S., \& Filaire, E. (2007). Eating attitudes, body esteem, perfectionism and anxiety of judo athletes and nonathletes. International Journal of Sports Medicine, 28(4), 340-345.

Sbriccoli, P., Camomilla, V., Di Mario, A., Quinzi, F., Figura, F., \& Felici, F. (2010). $\mathrm{N}$ euromuscular control adaptations in elite athletes: the case of top level karateka. European Journal Applied Physiology, 108(6), 1269-1280.

Schick, M. G., Brown, L. E., Coburn, J. W., Beam, W. C., Schick, E. E., \& Dabbs, N. C. (2010). Physiological Profile of Mixed Martial Artists. Medicina Sportiva, 14(4), 182-187.

Schmidt, W. D., Piencikowski, C. L., \& Vandervest, R. E. (2005). Effects of a competitive wrestling season on body composition, strength, and power in National Collegiate Athletic Association Division III college wrestlers. Journal of Strength and Conditioning Research, 19(3), 505-508.

Sertic , H., Segedi, I., \& Milanovic , D. (2006). Anthropological and fitness status of Croatian judoists. Archives of Budo, 2, 24-27.

Shephard, R. J. (1988). PAR-Q, Canadian Home Fitness Test and exercise screening alternatives. Sports M edicine, 5(3), 185-195.

Silva, J. J. R., Vecchio, F. B. D., Picanço, L. M., Takito, M. Y., \& Franchini, E. (2011). Time-Motion analysis in Muay-Thai and Kick-Boxing amateur matches. Journal of Human Sport and Exercise, 6(3), 1-7.

Sterkowicz-Przybycie, K. (2010). Technical diversification, body composition and somatotype of both heavy and light Polish jujitsukas of high level. Science and Sports, 25(4), 194-200.

Taylor, A. W., \& Brassard, L. (1981). A physiological profile of the Canadian judo team. Journal of Sports Medicine and Physical Fitness, 21(2), 160-164. 
Thomas, S. G., Cox, M. H., LeGal, Y. M., Verde, T. J., \& Smith, H. K. (1989). Physiological profiles of the Canadian National Judo Team. Canadian Journal of Sport Sciences, 14(3), 142-147.

Toskovic, N. N., Blessing, D., \& W illiford, H. N. (2002). The effect of experience and gender on cardiovascular and metabolic responses with dynamic Tae Kwon Do exercise. Journal of Strength and Conditioning Research, 16(2), 278-285.

Toskovic, N. N., Blessing, D., \& W illiford, H. N. (2004). Physiologic profile of recreational male and female novice and experienced Tae Kwon Do practitioners. Journal of Sports M edicine and Physical Fitness, 44(2), 164-172.

W orld Health Organization (2010). BMI classification. Disponible en http://apps. who.int/bmi/index.jsp?introPage=intro_3.html. [Consulta 11/29/ 2010].

Yoon, J. (2002). Physiological profiles of elite senior wrestlers. Sports Medicine, 32(4), 225-233. 